\title{
Relation of serum 25-hydroxyvitamin D status with skeletal muscle mass by sex and age group among Korean adults
}

\author{
Min Jung $\mathrm{Ko}^{1}$, Sungha Yun ${ }^{2}$, Kyungwon $\mathrm{Oh}^{2}$ and Kirang $\mathrm{Kim}^{3 *}$ \\ ${ }^{1}$ Division for Healthcare Technology Assessment Research, National Evidence-Based Healthcare Collaborating Agency, \\ Seoul, 100-705, Republic of Korea \\ ${ }^{2}$ Division of Health and Nutrition Survey, Korea Centers for Disease Control and Prevention, Chungbuk, 28160, Republic of Korea \\ ${ }^{3}$ Department of Food Science and Nutrition, Dankook University, Cheonan, 31116, Republic of Korea
}

(Submitted 22 June 2015 - Final revision received 29 July 2015 - Accepted 5 August 2015 - First published online 30 September 2015)

\section{Abstract}

The objective of this study was to examine whether high serum 25-hydroxyvitamin D (25(OH)D) concentration was associated with high skeletal muscle mass, taking into account the effects of sex and age among the participants of the Korea National Health and Nutrition Examination Survey (KNHANES) aged 40 years or older. This was a cross-sectional study using data from the 2009 to 2010 KNHANES; a total of 8406 subjects ( 3671 men and 4735 women) were included. The appendicular skeletal muscle mass index (ASMMI, $\mathrm{kg} / \mathrm{m}^{2}$ ) was estimated to measure the skeletal muscle mass. Hypovitaminosis was classified when the level of serum $25(\mathrm{OH}) \mathrm{D}$ was $<20 \mathrm{ng} / \mathrm{ml}$. The general linear model adjusted for confounding factors was used to determine differences in means of ASMMI by 25(OH)D status. The mean values of ASMMI were higher for men when compared with women. Women had a greater proportion of hypovitaminosis ( $71.1 \%)$ compared with men (53.2\%). After adjusting for multiple factors, men were seen to have significant differences in ASMMI based on 25(OH)D status regardless of age, showing a lower mean value of ASSMI in those with hypovitaminosis. However, there was no difference in ASMMI by 25(OH)D status among women in both younger and older age groups. In conclusion, we found that there might be a positive relationship between $25(\mathrm{OH}) \mathrm{D}$ and skeletal muscle mass in men, indicating that interventions to improve $25(\mathrm{OH}) \mathrm{D}$ levels that are aimed at increasing muscle mass could be beneficial for men with more rapid decreased rate of skeletal muscle mass.

\section{Key words: Vitamin D: Skeletal muscle mass: 25-Hydroxyvitamin D: Adults}

Ageing is associated with changes in body composition, generally with decreased weight and fat-free mass but increased fat mass ${ }^{(1-4)}$. The age-related loss of fat-free mass is of particular concern because it is related to a greater risk for functional impairment, disability, falls, impaired quality of life and mortality $^{(4-6)}$. In particular, loss of appendicular skeletal muscle mass (ASMM) is one of the components in the assessment of sarcopenia as a geriatric syndrome ${ }^{(7,8)}$. Sarcopenia is defined as the loss of muscle mass and strength with ageing and can increase the risk for functional limitation and mortality ${ }^{(6)}$. According to the results of the 2008-2009 Korea National Health and Nutrition Examination Survey (KNHANES), the prevalence of sarcopenia (class II) within the Korean population aged 40 years or older was $9.3 \%$ among men and $7.6 \%$ among women, when using a weight-adjusted definition ${ }^{(9)}$.

The decline in skeletal muscle mass associated with ageing has been known to depend on modifiable behavioural factors, such as diet and physical activity ${ }^{(8,10)}$. In particular, under-nutrition and the consumption of a less-varied diet with ageing ${ }^{(11)}$ are associated with less muscle mass. Among diet-related factors, a low vitamin D level has been known to influence declined muscle function and increase the risk of falling in the elderly ${ }^{(12,13)}$.

However, previous studies have reported an inconsistent relationship between vitamin $\mathrm{D}$ and muscle mass, in different sexes and age groups ${ }^{(14-20)}$. In addition, there is a lack of studies on the relationship between vitamin D and muscle mass with consideration of both sex and age. The prevalence of vitamin $\mathrm{D}$ deficiency and the extent of loss of skeletal muscle mass were reported to be different between sexes, showing a higher prevalence of vitamin D deficiency among women compared with men ${ }^{(21-23)}$ and a more rapid decreased rate of skeletal muscle mass after the age of 40 years in men compared with women ${ }^{(9,24-25)}$. Given the age- and sex-specific findings on vitamin D status and skeletal muscle mass, the relationship between vitamin $\mathrm{D}$ and muscle mass could be dependent on age and sex.

Abbreviations: 25(OH)D, 25-hydroxyvitamin D; ASMMI, appendicular skeletal muscle mass index; KNHANES, Korea National Health and Nutrition Examination Survey.

* Corresponding author: K. Kim, fax +82 41559 7857, email kirangkim@dankook.ac.kr 
Therefore, this study aimed to examine whether a high serum 25-hydroxyvitamin D (25(OH)D) concentration was related to a high skeletal muscle mass, taking into account the effects of sex and age among the national representative subjects who were 40 years or older. The hypotheses were that high serum $25(\mathrm{OH}) \mathrm{D}$ concentration is related to high skeletal muscle mass, and the difference in skeletal muscle mass by serum $25(\mathrm{OH}) \mathrm{D}$ status could be dependent on age and sex.

\section{Methods \\ Data sets and study participants}

This was a cross-sectional study using data from the 2009 to 2010 KNHANES. The KNHANES has been conducted periodically since 1998 to assess the health and nutrition status study conducted by the Korea Centers for Disease Control and Prevention (KCDC). Subjects younger than 40 years of age were excluded for better comparability while examining the relationship between $25(\mathrm{OH}) \mathrm{D}$ and skeletal muscle mass. The included subjects were grouped under the following age groups: (1) 40-64 years and (2) 65 years and older. The total number of subjects who were 40 years or older in the KNHANES was 9800; subjects without dual-energy X-ray absorptiometry (DXA) data, anthropometric data or vitamin $\mathrm{D}$ values were excluded ( $n$ 1394). Data from 8406 subjects (3671 men and 4735 women) were finally analysed in this cross-sectional study, which included $85.8 \%$ of the KNHANES population. All of the study participants provided informed consent, and the Institutional Review Board of the KCDC approved the study protocol.

\section{Serum 25-hydroxyvitamin D assessment}

Blood samples were collected from the subjects who had undertaken a more than 8-h overnight fast, immediately refrigerated, transported in cold storage to the Central Testing Institution in Seoul, Korea, and analysed within $24 \mathrm{~h}$. Serum 25(OH)D concentrations were measured with RIA (DiaSorin Inc.) using a counter (1470 Wizard; PerkinElmer). Participants were classified as having hypovitaminosis if the level of serum $25(\mathrm{OH}) \mathrm{D}$ was $<20 \mathrm{ng} / \mathrm{ml}^{(26)}$.

\section{Appendicular skeletal muscle mass assessment}

Whole-body fat-free mass $(\mathrm{kg})$ excluding the skeleton was based on the whole-body DXA scan using fan beam technology (Hologic). The muscle mass was estimated by measuring the appendicular skeletal muscle mass index (ASMMI, kg), which is one of the indices to identifying sarcopenia ${ }^{(27,28)}$ and is defined as the sum of the body mass (kg) from the arms and legs, based on the method of Heymsfield et al. ${ }^{(29)}$. Because absolute muscle mass is correlated with height, the relative muscle mass was considered so that the height-adjusted ASMMI was regarded as an index of relative skeletal muscle mass ${ }^{(27)}$. In this study, the ASMMI $\left(\mathrm{kg} / \mathrm{m}^{2}\right)$ defined as ASMM ( $\mathrm{kg}$ ) divided by height squared $\left(\mathrm{m}^{2}\right)$ was used as an outcome.

\section{Covariate assessment}

Information on socio-demographics and health-related behaviour was collected by the trained interviewers or self-reported. On the basis of education the subjects were categorised as follows: less than elementary school; middle school; high school; and college degree or higher. On the basis of occupation, subjects were categorised into the following groups: non-manual; manual; and no job. The income level was divided into quartiles based on the equivalent income of the household (monthly household income $/ \sqrt{\text { number of }}$ a household member). Information on history of diabetes, osteoarthritis, rheumatoid arthritis, myocardial infarction, angina, chronic obstructive pulmonary disease, tuberculosis, stroke and cancer was provided by the participants. Smoking and drinking statuses were categorised into current, previous and never. Participants were also asked whether they had taken dietary supplements for 2 weeks or more in the previous year. Status regarding marriage, menopausal status and year of participation in the survey were also examined. The participants' physical activity status was also classified into groups based on whether they met or did not meet the recommended criteria for physical activity: (1) $20 \mathrm{~min}$ or more/session of vigorous-intensity activity $3 \mathrm{~d}$ or more/week; or (2) $30 \mathrm{~min}$ or more/session of moderate-intensity physical activity $5 \mathrm{~d}$ or more/week.

BMI was calculated as weight $/$ height $^{2}\left(\mathrm{~kg} / \mathrm{m}^{2}\right)$. Measurement of intact parathyroid hormone (PTH) was carried out in the same laboratory using a chemiluminescence assay (DiaSorin).

Dietary intakes were assessed on the basis of a $24-\mathrm{h}$ recall in the nutrition survey. Daily intakes of total energy, carbohydrate, fat, protein, antioxidants (vitamin A and vitamin C) and $\mathrm{Ca}$, which is known to relate to skeletal muscle mass and $25(\mathrm{OH}) \mathrm{D}^{(30)}$, were estimated from the consumed food items.

\section{Statistical analysis}

For the representativeness of the study sample, the excluded samples were included in the data sets, and, after stratification, sample weights were assigned to a stratified group. Values were presented as mean values with their SE or as percentage with SE because the values were based on KNHANES-supplied sample weights and adjusted for age. Either the Student's $t$ test or the $\chi^{2}$ test was applied to evaluate the sex differences for general characteristics. The ASMMI was depicted by sex, age group and vitamin D status, all of which were examined by the $\chi^{2}$ test. To explore the factors related to vitamin D status, the participants' characteristics were compared according to their vitamin D status and the age group for each sex, using the Student's $t$ test or the $\chi^{2}$ test. The difference in ASMMI due to 25(OH)D status $(<20$ and $\geq 20 \mathrm{ng} / \mathrm{ml}$ ) was assessed by the general linear model, and the results were presented as least squares means after adjusting for confounding factors. The confounding factors that are related to lean body mass and serum 25(OH)D level were selected on the basis of a literature review. Only those confounding factors with a statistically significant relationship with $25(\mathrm{OH}) \mathrm{D}$ status within each sex and age group were included in the model. Regarding the nutrient variables, protein was highly correlated with carbohydrate and fat and hence it 
was excluded from the model because of multicollinearity problems. The analysis was stratified by sex and age groups. All of the analyses were weighted to allow for oversampling, nonresponse and the Korean population in the survey period, considering the complex sampling design. A type I error rate of 0.05 was used to validate the tests of hypothesis, and all analyses were performed using SAS version 9.2 (SAS Inc.).

\section{Results}

The general characteristics of the study participants, according to sex, are shown in Table 1. Among those who were 40-64 years of age, $81.5 \%$ were men and $75.9 \%$ were women. The mean value of ASMMI was higher among men and younger adults than among women and older adults. The difference in
ASMMI by age group was greater in men than in women. Women had a greater proportion of hypovitaminosis $(71.1 \%)$ compared with men $(53.2 \%)$, whereas the mean value of $25(\mathrm{OH}) \mathrm{D}$ was lower for women than for men (women $17.4 \mathrm{ng} / \mathrm{ml}$, men $20.3 \mathrm{ng} / \mathrm{ml}$ ). The proportion of graduates with college education or higher, that of smokers, drinkers and those engaged in manual jobs and the proportion of subjects engaged in regular exercise were higher among men than among women. Women had higher proportions of disease history and dietary supplement intake compared with men.

The factors related to $25(\mathrm{OH}) \mathrm{D}$ status, according to sex and age groups, are presented in Table 2. Most of the participants who had a low $25(\mathrm{OH}) \mathrm{D}(<20 \mathrm{ng} / \mathrm{ml})$ were, in higher proportions, commonly from the groups who had non-manual jobs, who were physically inactive, and whose participation season was spring or winter; their PTH values were also higher,

Table 1. Age-adjusted characteristics of the study subjects

(Mean values with their standard errors; percentages)

\begin{tabular}{|c|c|c|c|c|c|}
\hline & \multicolumn{2}{|c|}{ Men $(n$ 3671) } & \multicolumn{2}{|c|}{ Women (n 4735) } & \multirow[b]{2}{*}{$P$} \\
\hline & Mean & SE & Mean & SE & \\
\hline Age (mean, years) & $54 \cdot 3$ & 0.2 & $55 \cdot 7$ & 0.3 & $<0.0001$ \\
\hline \multicolumn{6}{|l|}{ Age group $(\%)$} \\
\hline 40-64 years & 81.5 & 0.8 & $75 \cdot 9$ & 0.9 & $<0.0001$ \\
\hline 65 years+ & $18 \cdot 5$ & 0.8 & $24 \cdot 1$ & 0.9 & \\
\hline Height (cm) & $168 \cdot 3$ & 0.1 & $155 \cdot 3$ & 0.1 & $<0.0001$ \\
\hline Weight $(\mathrm{kg})$ & $68 \cdot 4$ & 0.2 & $58 \cdot 0$ & 0.2 & $<0.0001$ \\
\hline BMI (mean, $\left.\mathrm{kg} / \mathrm{m}^{2}\right)$ & $24 \cdot 1$ & 0.1 & $24 \cdot 0$ & 0.1 & 0.3360 \\
\hline Whole-body fat-free mass (mean, $\mathrm{kg}$ ) & $52 \cdot 3$ & $0 \cdot 1$ & $37 \cdot 8$ & 0.1 & $<0.0001$ \\
\hline Whole-body fat-free mass except for skeleton (mean, $\mathrm{kg}$ ) & $49 \cdot 7$ & $0 \cdot 1$ & $35 \cdot 8$ & 0.1 & $<0.0001$ \\
\hline ASMM (mean, $\mathrm{kg})^{\star}$ & 22.9 & 0.1 & $15 \cdot 3$ & 0.0 & $<0.0001$ \\
\hline Percentage of ASMM (mean)† & $80 \cdot 1$ & $0 \cdot 2$ & 64.5 & $0 \cdot 1$ & $<0.0001$ \\
\hline ASMMI (mean, $\left.\mathrm{kg} / \mathrm{m}^{2}\right) \ddagger$ & 8.06 & 0.02 & $6 \cdot 32$ & 0.02 & $<0.0001$ \\
\hline ASMMI for 40-64 years & $8 \cdot 18$ & 0.02 & $6 \cdot 33$ & 0.02 & $<0.0001$ \\
\hline ASMMI for 65 years+ & 7.57 & 0.03 & $6 \cdot 24$ & 0.03 & $<0.0001$ \\
\hline Serum 25(OH)D (mean, ng/ml) & $20 \cdot 3$ & 0.2 & $17 \cdot 4$ & $0 \cdot 2$ & $<0.0001$ \\
\hline Hypovitaminosis D (\%)§ & $53 \cdot 2$ & 1.6 & $71 \cdot 1$ & 1.3 & $<0.0001$ \\
\hline \multicolumn{6}{|l|}{ Education level (\%) } \\
\hline$\leq$ Elementary school diploma & $22 \cdot 8$ & 0.9 & $40 \cdot 2$ & 0.8 & $<0.0001$ \\
\hline Middle school diploma & $16 \cdot 3$ & 0.8 & $15 \cdot 6$ & 0.7 & $<0.0001$ \\
\hline High school diploma & 33.5 & $1 \cdot 1$ & $30 \cdot 1$ & 0.8 & 0.0092 \\
\hline$\geq$ College diploma & $27 \cdot 4$ & $1 \cdot 2$ & $14 \cdot 1$ & 0.8 & $<0.0001$ \\
\hline \multicolumn{6}{|l|}{ Occupation (\%) } \\
\hline Non-manual & $23 \cdot 2$ & $1 \cdot 1$ & $9 \cdot 7$ & 0.5 & $<0.0001$ \\
\hline Manual & $56 \cdot 1$ & $1 \cdot 3$ & $42 \cdot 1$ & $1 \cdot 1$ & $<0.0001$ \\
\hline No job & $20 \cdot 7$ & 0.9 & $48 \cdot 2$ & 1.0 & $<0.0001$ \\
\hline Disease history (\%)\| & $27 \cdot 4$ & 0.8 & 35.5 & 0.8 & $<0.0001$ \\
\hline \multicolumn{6}{|l|}{ Smoking status (\%) } \\
\hline Current & $42 \cdot 0$ & 1.0 & $5 \cdot 2$ & 0.5 & $<0.0001$ \\
\hline Previous & $40 \cdot 3$ & 1.0 & 2.5 & 0.3 & $<0.0001$ \\
\hline Never & $17 \cdot 7$ & 0.7 & $92 \cdot 3$ & 0.5 & $<0.0001$ \\
\hline \multicolumn{6}{|l|}{ Drinking status (\%) } \\
\hline Current & $82 \cdot 0$ & 0.7 & $60 \cdot 2$ & 0.9 & $<0.0001$ \\
\hline Previous & 11.5 & 0.5 & $15 \cdot 2$ & 0.6 & $<0.0001$ \\
\hline Never & 6.5 & 0.5 & $24 \cdot 6$ & 0.7 & $<0.0001$ \\
\hline Taking dietary supplements (\%)ף & $31 \cdot 1$ & $1 \cdot 0$ & $48 \cdot 2$ & 1.0 & $<0.0001$ \\
\hline Physical activity $(\%)^{* \star}$ & $26 \cdot 7$ & 1.0 & $23 \cdot 7$ & 0.9 & 0.0066 \\
\hline
\end{tabular}

ASMM, appendicular skeletal muscle mass; ASMMI, ASMM index; 25(OH)D, 25-hydroxyvitamin D (ng/ml).

* ASMM: lean body mass $(\mathrm{kg})$ of arms and legs.

$\dagger$ Percentage of ASMM: lean body mass $(\mathrm{kg})$ of arms and legs/total weight of arms and legs $\times 100$.

$\ddagger$ ASMMI $\left(\mathrm{kg} / \mathrm{m}^{2}\right)$ : ASMM $(\mathrm{kg}) /$ height $(\mathrm{m})^{2}$.

$\S$ Hypovitaminosis $\mathrm{D} ; 20 \mathrm{ng} / \mathrm{ml}<$ serum $25-(\mathrm{OH}) \mathrm{D}$.

$\|$ History of diabetes, osteoarthritis, rheumatoid arthritis, myocardial infarction, angina, chronic obstructive pulmonary disease, tuberculosis, stroke and cancer.

II Taking dietary supplements for 2 weeks or more during the past 1 year.

** $20 \mathrm{~min}$ or more/session of vigorous-intensity activity $3 \mathrm{~d}$ or more/week, or $30 \mathrm{~min}$ or more/session of moderate-intensity physical activity $5 \mathrm{~d}$ or more/week. 
Table 2. Age-adjusted mean values of factors related to 25 -hydroxyvitamin $\mathrm{D}(25(\mathrm{OH}) \mathrm{D})$ status by sex and age group (Mean values with their standard errors; percentages)

\begin{tabular}{|c|c|c|c|c|c|c|c|c|c|c|c|c|c|c|c|c|c|c|c|c|}
\hline & \multicolumn{10}{|c|}{ Men $(n$ 3671) } & \multicolumn{10}{|c|}{ Women ( $n$ 4735) } \\
\hline & \multicolumn{5}{|c|}{$40-64$ years $(n$ 2543) } & \multicolumn{5}{|c|}{65 years $+(n 1128)$} & \multicolumn{5}{|c|}{ 40-64 years $(n 3286)$} & \multicolumn{5}{|c|}{65 years $+(n 1449)$} \\
\hline & \multicolumn{2}{|c|}{$\begin{array}{l}<20 \mathrm{ng} / \mathrm{ml} \\
(n 1377)\end{array}$} & \multicolumn{2}{|c|}{$\begin{array}{c}\geq 20 \mathrm{ng} / \mathrm{ml} \\
(n 1166)\end{array}$} & \multirow[b]{2}{*}{$P^{\star}$} & \multicolumn{2}{|c|}{$\begin{array}{c}<20 \mathrm{ng} / \mathrm{ml} \\
(n 533)\end{array}$} & \multicolumn{2}{|c|}{$\begin{array}{c}\geq 20 \mathrm{ng} / \mathrm{ml} \\
(n 595)\end{array}$} & \multirow[b]{2}{*}{$P^{*}$} & \multicolumn{2}{|c|}{$\begin{array}{l}<20 \mathrm{ng} / \mathrm{ml} \\
(n 2360)\end{array}$} & \multicolumn{2}{|c|}{$\begin{array}{c}\geq 20 \mathrm{ng} / \mathrm{ml} \\
(n 926)\end{array}$} & \multirow[b]{2}{*}{$P^{*}$} & \multicolumn{2}{|c|}{$\begin{array}{c}<20 \mathrm{ng} / \mathrm{ml} \\
(n 912)\end{array}$} & $\begin{array}{r}\geq 20 \mathrm{n} \\
(n 53\end{array}$ & $\begin{array}{l}\mathrm{g} / \mathrm{ml} \\
37)\end{array}$ & \\
\hline & Mean & SE & Mean & SE & & Mean & SE & Mean & SE & & Mean & SE & Mean & SE & & Mean & $\mathrm{SE}$ & Mean & SE & $P^{*}$ \\
\hline Age (mean, years) & $49 \cdot 8$ & 0.2 & $50 \cdot 9$ & 0.2 & 0.0005 & 71.5 & 0.2 & 71.9 & 0.3 & 0.2636 & $49 \cdot 7$ & 0.2 & $52 \cdot 2$ & 0.3 & $<0.0001$ & 72.5 & 0.2 & $72 \cdot 6$ & 0.3 & 0.8949 \\
\hline Height (mean, $\mathrm{cm}$ ) & 169.5 & 0.2 & $169 \cdot 2$ & 0.2 & 0.4133 & $165 \cdot 3$ & 0.3 & 164.8 & 0.3 & 0.2034 & 156.6 & 0.1 & 156.6 & 0.2 & 0.9937 & $151 \cdot 2$ & 0.2 & $150 \cdot 2$ & 0.3 & 0.0086 \\
\hline Weight (mean, kg) & $70 \cdot 0$ & 0.3 & 69.8 & 0.3 & 0.6513 & 63.6 & 0.5 & 62.6 & 0.4 & 0.1101 & 58.6 & 0.2 & 58.7 & 0.4 & 0.8787 & $56 \cdot 3$ & 0.4 & $54 \cdot 3$ & 0.4 & 0.0008 \\
\hline $\mathrm{BMI}\left(\mathrm{mean}, \mathrm{kg} / \mathrm{m}^{2}\right)$ & $24 \cdot 3$ & 0.1 & 24.4 & 0.1 & 0.8094 & 23.2 & 1.6 & 23.0 & 0.1 & 0.3384 & 23.9 & 0.1 & 23.9 & 0.1 & 0.9858 & 24.6 & 0.1 & $24 \cdot 0$ & 0.2 & 0.0072 \\
\hline ASMM (mean, kg) & $23 \cdot 3$ & 0.1 & 23.7 & 0.1 & 0.0081 & $20 \cdot 3$ & 0.2 & 20.8 & 0.1 & 0.0111 & $15 \cdot 5$ & 0.1 & $15 \cdot 6$ & 0.1 & 0.2813 & 14.3 & 0.1 & $14 \cdot 2$ & 0.1 & 0.6826 \\
\hline ASMMI (mean, $\left.\mathrm{kg} / \mathrm{m}^{2}\right) \dagger$ & 8.10 & 0.03 & 8.27 & 0.03 & $<0.0001$ & 7.42 & 0.05 & 7.65 & 0.04 & $<0.0001$ & $6 \cdot 31$ & 0.02 & $6 \cdot 36$ & 0.03 & 0.1906 & $6 \cdot 23$ & 0.03 & $6 \cdot 28$ & 0.04 & 0.2904 \\
\hline PTH (mean) & 70.7 & 1.9 & 63.6 & 1.4 & 0.0002 & 73.8 & 1.9 & 64.6 & 1.2 & $<0.0001$ & 67.8 & 1.5 & $59 \cdot 2$ & 1.6 & $<0.0001$ & 79.9 & 1.5 & $68 \cdot 3$ & 1.6 & $<0.0001$ \\
\hline Education level (\%) & & & & & & & & & & & & & & & & & & & & \\
\hline$\leq$ Elementary school & $12 \cdot 6$ & $1 \cdot 1$ & 16.9 & $1 \cdot 2$ & 0.0034 & 44.4 & 2.5 & $56 \cdot 9$ & $2 \cdot 8$ & 0.0005 & $28 \cdot 3$ & 1.5 & 26.8 & 1.0 & 0.3847 & 86.7 & 1.5 & $87 \cdot 3$ & 1.8 & 0.7357 \\
\hline Middle & 14.9 & 1.1 & $17 \cdot 1$ & 1.3 & 0.1846 & $21 \cdot 0$ & $2 \cdot 2$ & $15 \cdot 2$ & 1.7 & 0.0311 & 18.5 & 1.0 & 18.7 & 1.5 & 0.9135 & 7.1 & 1.0 & 5.5 & 1.2 & 0.3139 \\
\hline High & 37.8 & 1.6 & 38.0 & 1.9 & 0.9607 & $23 \cdot 0$ & $2 \cdot 2$ & $15 \cdot 0$ & 1.7 & 0.0040 & 37.3 & $1 \cdot 2$ & $36 \cdot 3$ & $2 \cdot 0$ & 0.6458 & $5 \cdot 2$ & $1 \cdot 1$ & 4.9 & 1.0 & 0.8401 \\
\hline$\geq$ College & 34.7 & 1.9 & $28 \cdots 0$ & 1.8 & 0.0050 & 11.6 & 1.9 & $12 \cdot 9$ & $2 \cdot 1$ & 0.6327 & 17.4 & $1 \cdot 1$ & $16 \cdot 7$ & $1 \cdot 6$ & 0.7158 & $1 \cdot 0$ & 0.3 & $2 \cdot 3$ & 0.8 & 0.1642 \\
\hline Income (\%) & & & & & & & & & & & & & & & & & & & & \\
\hline Q1 (lowest) & $26 \cdot 0$ & 1.5 & 25.9 & 1.8 & 0.9726 & $22 \cdot 8$ & $2 \cdot 2$ & $24 \cdot 8$ & $2 \cdot 4$ & 0.5256 & $24 \cdot 8$ & $1 \cdot 3$ & $26 \cdot 1$ & $1 \cdot 8$ & 0.5110 & $23 \cdot 2$ & 1.9 & 28.9 & $2 \cdot 7$ & 0.0858 \\
\hline Q2 & 25.4 & 1.3 & $27 \cdot 3$ & 1.7 & 0.3890 & 22.5 & $2 \cdot 0$ & 25.5 & $2 \cdot 0$ & 0.2924 & $25 \cdot 3$ & $1 \cdot 1$ & 26.8 & 1.8 & 0.4495 & $24 \cdot 3$ & 1.7 & $22 \cdot 2$ & $2 \cdot 0$ & 0.4144 \\
\hline Q3 & $25 \cdot 3$ & 1.4 & $23 \cdot 0$ & 1.5 & 0.2350 & 26.8 & 2.4 & 24.9 & $2 \cdot 4$ & 0.5745 & 26.0 & $1 \cdot 1$ & 23.5 & 1.5 & 0.1786 & 24.9 & 1.7 & $24 \cdot 3$ & $2 \cdot 2$ & 0.8201 \\
\hline Q4 (highest) & $23 \cdot 3$ & 1.6 & 23.8 & 1.7 & 0.7649 & $27 \cdot 9$ & $2 \cdot 2$ & 24.8 & $2 \cdot 1$ & 0.2983 & 23.9 & $1 \cdot 3$ & 23.6 & 1.9 & 0.8654 & 27.6 & $2 \cdot 1$ & $24 \cdot 6$ & $2 \cdot 2$ & 0.2994 \\
\hline Occupation (\%) & & & & & & & & & & & & & & & & & & & & \\
\hline Non-manual & 33.0 & 1.6 & $23 \cdot 1$ & 1.7 & $<0.0001$ & 3.6 & 0.9 & $2 \cdot 2$ & 0.7 & 0.2163 & $12 \cdot 7$ & 0.8 & 9.4 & $1 \cdot 1$ & 0.0133 & 0.3 & 0.2 & 0.2 & 0.2 & 0.6619 \\
\hline Manual & $54 \cdot 3$ & 1.8 & $66 \cdot 5$ & 1.8 & $<0.0001$ & $31 \cdot 8$ & $2 \cdot 4$ & $50 \cdots 0$ & $3 \cdot 0$ & $<0.0001$ & $45 \cdot 3$ & 1.4 & 50.6 & $2 \cdot 5$ & 0.0518 & 23.6 & $2 \cdot 0$ & $31 \cdot 3$ & $2 \cdot 9$ & 0.0114 \\
\hline No job & $12 \cdot 7$ & $1 \cdot 0$ & $10 \cdot 4$ & $1 \cdot 1$ & 0.1065 & $64 \cdot 7$ & $2 \cdot 4$ & $48 \cdot 1$ & 0.3 & $<0.0001$ & $42 \cdot 0$ & $1 \cdot 3$ & $40 \cdot 0$ & $2 \cdot 2$ & 0.4089 & $76 \cdot 1$ & 1.9 & 68.4 & $2 \cdot 9$ & 0.0133 \\
\hline Disease history (\%) & $22 \cdot 7$ & 1.3 & 20.6 & 1.2 & 0.2309 & $52 \cdot 4$ & 2.5 & 41.9 & $2 \cdot 3$ & 0.0029 & 27.6 & $1 \cdot 0$ & 30.6 & 1.7 & 0.1710 & 61.5 & 1.9 & $62 \cdot 4$ & 2.6 & 0.7867 \\
\hline Current smoker (\%) & $46 \cdot 7$ & 1.6 & 45.5 & 1.7 & 0.5884 & 28.2 & $2 \cdot 3$ & $21 . .9$ & 1.8 & 0.0383 & 4.5 & 0.5 & $6 \cdot 0$ & $1 \cdot 2$ & 0.2415 & 5.5 & 0.9 & 2.9 & 0.9 & 0.0433 \\
\hline Current drinker (\%) & 83.9 & $1 \cdot 2$ & 89.6 & 1.0 & 0.0002 & $62 \cdot 2$ & $2 \cdot 3$ & $70 \cdot 0$ & $2 \cdot 3$ & 0.0187 & $65 \cdot 3$ & $1 \cdot 2$ & 71.8 & 1.8 & 0.0028 & 34.7 & $2 \cdot 1$ & 37.5 & 2.5 & 0.3835 \\
\hline Taking dietary supplements (\%) & $30 \cdot 2$ & 1.5 & $30 \cdot 2$ & 1.8 & 0.9822 & 30.5 & 2.5 & 37.2 & $2 \cdot 9$ & 0.0635 & 48.8 & $1 \cdot 3$ & 55.2 & $2 \cdot 1$ & 0.0092 & 38.8 & $2 \cdot 2$ & 44.1 & $2 \cdot 9$ & 0.1344 \\
\hline Physical activity (\%)‡ & $25 \cdot 1$ & 1.4 & 31.6 & 1.7 & 0.0027 & 19.0 & $2 \cdot 1$ & $24 \cdot 3$ & $2 \cdot 3$ & 0.0737 & 24.4 & $1 \cdot 1$ & 29.0 & $2 \cdot 1$ & 0.0415 & 15.5 & 1.6 & 20.9 & $2 \cdot 2$ & 0.0468 \\
\hline Survey season (\%) & & & & & & & & & & & & & & & & & & & & \\
\hline Spring & $32 \cdot 0$ & 3.2 & $16 \cdot 9$ & 2.5 & $<0.0001$ & $32 \cdot 0$ & $4 \cdot 0$ & $15 \cdot 9$ & 3.2 & $<0.0001$ & 29.0 & $2 \cdot 9$ & 12.5 & $2 \cdot 2$ & $<0.0001$ & 32.5 & 3.9 & 11.9 & $2 \cdot 6$ & $<0.0001$ \\
\hline Summer & $20 \cdot 1$ & 2.6 & 37.9 & 3.7 & $<0.0001$ & $17 \cdot 3$ & 2.6 & $30 \cdot 0$ & 4.1 & 0.0015 & 21.7 & $2 \cdot 4$ & 40.4 & 4.1 & $<0.0001$ & 18.4 & 2.6 & 33.0 & 4.7 & 0.0005 \\
\hline Fall & $16 \cdot 3$ & $2 \cdot 2$ & $27 \cdot 4$ & 3.2 & $<0.0001$ & 20.7 & 3.8 & 33.6 & 4.4 & 0.0050 & 20.2 & $2 \cdot 5$ & 29.1 & 3.5 & 0.0012 & 21.8 & 3.1 & 37.8 & 4.8 & 0.0002 \\
\hline Winter & $31 \cdot 6$ & $3 \cdot 3$ & $17 \cdot 8$ & $2 \cdot 6$ & $<0.0001$ & $30 \cdot 0$ & 3.9 & 20.5 & 3.6 & 0.0188 & 29.1 & 3.0 & $18 \cdot 0$ & $2 \cdot 7$ & $<0.0001$ & $27 \cdot 3$ & 3.5 & $17 \cdot 3$ & 3.4 & 0.0036 \\
\hline Postmenopausal (\%) & - & - & - & & - & - & - & - & & - & 49.3 & 0.9 & $54 \cdot 0$ & 1.3 & 0.0027 & 100 & & 100 & & - \\
\hline Nutrient intake (mean, per d) & & & & & & & & & & & & & & & & & & & & \\
\hline Energy $(\mathrm{kJ})$ & $9363 \cdot 8$ & $101 \cdot 7$ & $9577 \cdot 1$ & $112 \cdot 1$ & $0 \cdot 1461$ & $7485 \cdot 2$ & $113 \cdot 8$ & $8204 \cdot 8$ & 125.9 & $<0.0001$ & $6970 \cdot 5$ & $61 \cdot 1$ & 7263.4 & 109.6 & 0.0148 & $6058 \cdot 4$ & $92 \cdot 0$ & $6112 \cdot 8$ & 117.8 & 0.7125 \\
\hline Energy (kcal) & 2238 & $24 \cdot 3$ & 2289 & $26 \cdot 8$ & 0.1461 & 1789 & $27 \cdot 2$ & 1961 & $30 \cdot 1$ & $<0.0001$ & 1666 & 14.6 & 1736 & $26 \cdot 2$ & 0.0148 & 1448 & $22 \cdot 0$ & 1461 & 28.2 & 0.7125 \\
\hline Carbohydrate (g) & 355 & $4 \cdot 1$ & 359 & 4.6 & 0.4615 & 319 & 4.7 & 336 & $5 \cdot 2$ & 0.0086 & 289 & 2.5 & 305 & $5 \cdot 1$ & 0.0020 & 280 & 4.4 & 281 & 5.5 & 0.8910 \\
\hline Fat $(\mathrm{g})$ & $42 \cdot 0$ & 0.8 & 41.7 & $1 \cdot 1$ & 0.7982 & $25 \cdot 3$ & 0.9 & 28.8 & $1 \cdot 2$ & 0.0158 & 30.5 & 5.9 & 31.1 & 0.8 & 0.6001 & $17 \cdot 2$ & 0.6 & $18 \cdot 1$ & 0.7 & 0.3434 \\
\hline Protein (g) & $80 \cdot 7$ & 1.2 & 82.7 & 1.5 & 0.2677 & $60 \cdot 0$ & 1.5 & 65.5 & 1.5 & 0.0077 & 59.7 & 0.7 & 61.5 & $1 \cdot 1$ & 0.1623 & 44.8 & 0.9 & 45.9 & 1.0 & 0.4037 \\
\hline $\mathrm{Ca}(\mathrm{g})$ & 577 & 10.5 & 589 & $12 \cdot 3$ & 0.4171 & 491 & 21.4 & 500 & $17 \cdot 3$ & 0.7330 & 474 & 8.8 & 489 & $10 \cdot 7$ & 0.2466 & 373 & $25 \cdot 4$ & 383 & $15 \cdot 3$ & 0.7500 \\
\hline Vitamin A ( $\mu \mathrm{g} R E$ ) & 887 & 29.9 & 935 & 38.5 & 0.3183 & 620 & 35.9 & 733 & 40.3 & 0.0378 & 804 & $27 \cdot 2$ & 855 & 40.5 & 0.2689 & 614 & 50.5 & 566 & 37.0 & 0.4438 \\
\hline Vitamin C (mg) & 119 & 3.0 & 115 & 2.9 & 0.2498 & 88.4 & 3.9 & 93.5 & 3.7 & 0.3408 & 111 & 2.6 & 107 & 3.6 & 0.3227 & 75.7 & 3.3 & 76.4 & 2.9 & 0.8773 \\
\hline
\end{tabular}

ASMM, appendicular skeletal muscle mass; ASMMI, ASMM index; PTH, parathyroid hormone; Q, quintile; RE, retinol equivalents.

* $P$ value was calculated using the Student's $t$ test or the $x^{2}$ test

† ASMMI $\left(\mathrm{kg} / \mathrm{m}^{2}\right)=$ weight of ASMM $(\mathrm{kg}) /$ height $(\mathrm{m})^{2}$

₹ Physical activity $=20 \mathrm{~min}$ or more/session of vigorous-intensity activity $3 \mathrm{~d}$ or more/week, or $30 \mathrm{~min}$ or more/session of moderate-intensity physical activity $5 \mathrm{~d}$ or more/week. 
Table 3. The difference in appendicular skeletal muscle mass index between 25 -hydroxyvitamin $D$ status $(<20$ and $\geq 20 \mathrm{ng} / \mathrm{ml})$ by sex and age group

(Mean values with their standard errors)

\begin{tabular}{|c|c|c|c|c|c|}
\hline & \multirow{2}{*}{\multicolumn{2}{|c|}{$\frac{<20 \mathrm{ng} / \mathrm{ml}}{(n \text { 1377) }}$}} & \multirow{2}{*}{\multicolumn{2}{|c|}{$\frac{\geq 20 \mathrm{ng} / \mathrm{ml}}{(n 1166)}$}} & \multirow[b]{3}{*}{$P \ddagger$} \\
\hline & & & & & \\
\hline & Mean & SE & Mean & SE & \\
\hline \multicolumn{6}{|c|}{ Men (years) } \\
\hline $40-64^{*}$ & 8.09 & 0.07 & $8 \cdot 24$ & 0.07 & 0.0037 \\
\hline $65 \dagger$ & 7.55 & 0.05 & $7 \cdot 70$ & 0.06 & 0.0175 \\
\hline \multicolumn{6}{|c|}{ Women (years) } \\
\hline 40-64‡ & $6 \cdot 35$ & 0.05 & $6 \cdot 41$ & 0.05 & 0.1677 \\
\hline $65 \S$ & $6 \cdot 28$ & 0.03 & $6 \cdot 35$ & 0.04 & 0.0624 \\
\hline
\end{tabular}

* The model was adjusted for age, parathyroid hormone (PTH), educational level, occupation, current drinking status, physical activity and survey season.

† The model was adjusted for age, PTH, educational level, occupation, disease history, current smoking status, current drinking status, taking dietary supplements, survey season, energy, carbohydrate and fat.

‡ The model was adjusted for age, PTH, occupation, current drinking status, taking dietary supplements, survey season, postmenopausal status, energy and carbohydrate.

$\S$ The model was adjusted for age, BMI, PTH, occupation, current smoking status and survey season.

regardless of sex or age group, when compared with those with a high $25(\mathrm{OH})$ D level $(\geq 20 \mathrm{ng} / \mathrm{ml})$. In men with a low $25(\mathrm{OH}) \mathrm{D}$ level, the mean value of ASMMI was lower and the education level was higher compared with men who had a high $25(\mathrm{OH}) \mathrm{D}$ level. The average intakes of energy in subjects with a low 25(OH)D were lower in men aged 65 years and above and in women aged 40-64 years when compared with those with a high $25(\mathrm{OH}) \mathrm{D}$. There was a higher proportion of current smokers among participants aged 65 years and older who had a low $25(\mathrm{OH}) \mathrm{D}$ level compared with those with a high 25(OH)D

After adjusting for confounding factors, a positive relationship between 25(OH)D and ASMMI was seen in men but not in women (Table 3). There was a significant difference in ASMMI based on 25(OH)D status in men regardless of age, showing a lower mean value of ASSMI in those with hypovitaminosis (8.09 for $<20 \mathrm{ng} / \mathrm{ml}$ of $25(\mathrm{OH}) \mathrm{D}$ and 8.24 for $\geq 20 \mathrm{ng} / \mathrm{ml}$ among those aged 40-64 years, $P$ value 0.0037 ; and 7.55 for $<20 \mathrm{ng} / \mathrm{ml}$ of 25 $(\mathrm{OH}) \mathrm{D}$ and 7.70 for $\geq 20 \mathrm{ng} / \mathrm{ml}$ among those aged 65 years or more, $P$ value 0.0175$)$. However, there was no difference among women in both the younger and older age groups.

\section{Discussion}

Given the inconsistent findings for the relationship between $25(\mathrm{OH}) \mathrm{D}$ and skeletal muscle mass, we aimed to study the relationship between $25(\mathrm{OH}) \mathrm{D}$ and skeletal muscle mass by sex and age group in the Korean adult population. We found that there was a positive relationship between 25(OH)D level and skeletal muscle mass in both young and old age groups among men.

Several studies have addressed the mechanism of how vitamin D may relate to the skeletal muscle. Evidence has indicated that the receptor for 1,25-dihydroxyvitamin D
(1,25(OH)2D), vitamin D receptor (VDR), is expressed in skeletal muscle and is a crucial mediator of $1,25(\mathrm{OH}) 2 \mathrm{D}$, affecting muscle contractility ${ }^{(31)}$. However, the result is still inconsistent as shown by another study in which the VDR was undetectable in skeletal, cardiac and smooth muscle ${ }^{(32)}$. The vitamin $\mathrm{D}$ deficiency might affect muscle protein turnover by inducing hypocalcaemia and decreasing insulin secretion ${ }^{(33)}$. Vitamin D metabolites have been found to affect muscle metabolism in three ways: (1) by mediating gene transcription; (2) through rapid pathways not involving DNA synthesis; and (3) by the allelic variant of the $\mathrm{VDR}^{(20)}$.

The skeletal muscle can experience functional improvement over time; the effect of $25(\mathrm{OH}) \mathrm{D}$ on the skeletal muscle mass could be important for improving muscle-related outcomes. However, most of the studies that examined the effects of 25(OH)D on muscle-related outcomes were focused on muscle function and performance ${ }^{(15,34)}$. Moreover, the effects of $25(\mathrm{OH}) \mathrm{D}$ on the skeletal muscle mass were mostly evaluated on the elderly population ${ }^{(18-20,35,36)}$, indicating a lack of data on the effects of $25(\mathrm{OH}) \mathrm{D}$ on outcome measures in younger adults. Even for the elderly population, the findings on the effect of $25(\mathrm{OH}) \mathrm{D}$ on the skeletal muscle mass were inconsistent, showing either a positive ${ }^{(18,19,20,36)}$ effect or no effect ${ }^{(18,37)}$. In terms of inconsistent findings, several studies explained that the reason for the absence of effects of $25(\mathrm{OH}) \mathrm{D}$ on the muscle mass could be that $25(\mathrm{OH}) \mathrm{D}$ might affect the coordinative muscle function more than it does muscle mass or strength. A systematic review or a meta-analysis of randomised controlled trials showed that supplemental vitamin D improved either the physical functioning or reduced the risk of falls ${ }^{(15,34)}$.

Although this study indicated that $25(\mathrm{OH}) \mathrm{D}$ was positively related to skeletal muscle mass in men regardless of age group, there were no consistent results by sex. Shantavasinkul et al. ${ }^{(38)}$ found that $25(\mathrm{OH}) \mathrm{D}$ levels have a positive relationship with a marker of muscle mass in older men. In contrast, a recent study on the effect of $25(\mathrm{OH}) \mathrm{D}$ on the muscle mass reported that only older women had lower skeletal muscle mass associated with lower 25(OH)D levels ${ }^{(18)}$.

A possible explanation for the difference due to sex could be the effect of the genotype of VDR (Fok1) on the muscle mass $^{(31)}$. The risk allele ( $F$ allele) that would be associated with reduced muscle mass was differently distributed in each sex and ethnicity. Women had a lower proportion of the risk allele compared with men and, among ethnic groups, Asian groups, such as the Japanese, had the lowest proportion of the risk allele than did UK, French and North Indians ${ }^{(39)}$. In this study on the Korean population, a positive relationship between 25(OH)D and skeletal muscle mass in men might be explained by the characteristic of men having a higher proportion of the risk allele. Given that men have a relatively more rapid decreased rate of skeletal muscle mass after 40 years, it could be important to maintain their serum 25(OH)D levels in the normal range. Further investigation on the ethnicity- and sex-specific effects of serum $25(\mathrm{OH}) \mathrm{D}$ on muscle mass in additional diverse populations of adults is needed to better understand the mechanism of these findings.

Several limitations should be considered while interpreting the findings of our study. First, the cross-sectional nature of this 
study did not allow for the assessment of the causal relationship between $25(\mathrm{OH}) \mathrm{D}$ levels and lean body mass. Second, this study was conducted year round. As season may affect the 25(OH)D levels and muscle mass at the same time, season was adjusted in all analyses to minimise the effect from seasonal variation. Third, vitamin D measured at tissue levels might be more sensitive to muscle mass than that measured at serum levels; these findings had some discrepancies ${ }^{(32)}$. Despite the abovementioned limitations, this study included a representative population-based sample with a large number of adults, including younger adults, and studied the relationship between serum 25 $(\mathrm{OH}) \mathrm{D}$ and skeletal muscle mass, considering age and sex.

In conclusion, in this representative population-based sample of Korean adults of varying ages, there might be a positive relationship between serum $25(\mathrm{OH}) \mathrm{D}$ concentration and skeletal muscle mass among men regardless of age, indicating that the interventions to improve $25(\mathrm{OH}) \mathrm{D}$ levels aimed at increasing muscle mass could be beneficial for men with more rapid decreased rate of skeletal muscle mass.

\section{Acknowledgements}

This work was supported by Basic Science Research Program through the National Research Foundation of Korea (NRF) funded by the Ministry of Science, ICT \& Future Planning (no. 2013R1A1A1060704). This work was supported by the National Evidence-based Healthcare Collaborating Agency.

M. J. K. designed the study, contributed to data interpretation and wrote the manuscript. S. Y. analysed the data. K. O. contributed to data interpretation and assisted with statistical analyses. K. K. designed the study, contributed to data interpretation and supervised all aspects of its implementation. All authors read and approved the final manuscript.

None of the authors has any conflicts of interest.

\section{References}

1. Forbes GB \& Reina JC (1970) Adult lean body mass declines with age: some longitudinal observations. Metabolism 19, 653-663.

2. Kyle UG, Genton L, Slosman DO, et al. (2001) Fat-free and fat mass percentiles in 5225 healthy subjects aged 15 to 98 years. Nutrition 17, 534-541.

3. Hughes VA, Frontera WR, Roubenoff R, et al. (2002) Longitudinal changes in body composition in older men and women: role of body weight change and physical activity. Am J Clin Nutr 76, 473-481.

4. Genton L, Karsegard VL, Chevalley T, et al. (2011) Body composition changes over 9 years in healthy elderly subjects and impact of physical activity. Clin Nutr 30, 436-442.

5. Bales CW \& Ritchie CS (2002) Sarcopenia, weight loss, and nutritional frailty in the elderly. Annu Rev Nutr 22, 309-323.

6. Janssen I, Heymsfield SB \& Ross R (2002) Low relative skeletal muscle mass (sarcopenia) in older persons is associated with functional impairment and physical disability. $J$ Am Geriatr Soc 50, 889-896.

7. Dutta C (1997) Significance of Sarcopenia in the Elderly. J Nutr 127, 992S-993S.

8. Cruz-Jentoft AJ, Baeyens JP, Bauer JM, et al. (2010) Sarcopenia: European consensus on definition and diagnosis. Age Ageing 39, 412-423.
9. Kim YS, Lee Y, Chung YS, et al. (2012) Prevalence of sarcopenia and sarcopenic obesity in the Korean population based on the Fourth Korean National Health and Nutritional Examination Surveys. J Gerontol A Biol Sci Med Sci 67, 1107-1113.

10. Scott D, Blizzard L, Fell J, et al. (2011) The epidemiology of sarcopenia in community living older adults: what role does lifestyle play? J Cachexia Sarcopenia Muscle 2, 125-134.

11. Fox T, Brummit PS \& Ferguson-Wolf M (2000) Position of the American dietetic association: nutrition, aging, and the continuum of care. J Am Diet Assoc 100, 580-595.

12. Bischoff-Ferrari HA, Borchers M, Gudat F, et al. (2004) Vitamin D receptor expression in human muscle tissue decreases with age. J Bone Miner Res 19, 265-269.

13. Hamilton B (2010) Vitamin D and human skeletal muscle. Scand J Med Sci Sports 20, 182-2190.

14. Verreault R, Semba R, Volpato D, et al. (2002) Low serum vitamin $\mathrm{D}$ does not predict new disability or loss of muscle strength in older women. J Am Geriatr Soc 50, 912-917.

15. Annweiler C, Beauchet O, Berrut G, et al. (2009) Is there an association between serum 25-hydroxyvitamin D concentration and muscle strength among older women? Results from baseline assessment of the EPIDOS study. J Nutr Health Aging 13, 90-95.

16. Ceglia L, Chiu GR, Harris SS, et al. (2011) Serum 25-hydroxyvitamin D concentration and physical function in adult men. Clin Endocrinol (Oxf) 74, 370-376.

17. Gilsanz V, Kremer A, Mo AO, et al. (2010) Vitamin D status and its relation to muscle mass and muscle fat in young women. J Clin Endocrinol Metab 95, 1595-1601.

18. Iannuzzi-Sucich M, Prestwood KM \& Kenny AM (2002) Prevalence of sarcopenia and predictors of skeletal muscle mass in healthy, older men and women. J Gerontol A Biol Sci Med Sci 57, M772-M777.

19. Scott D, Blizzard L, Fell J, et al. (2010) A prospective study of the associations between 25-hydroxy-vitamin D, sarcopenia progression and physical activity in older adults. Clin Endocrinol (Oxf) 73, 581-587.

20. Visser M, Deeg DJ \& Lips P (2003) Low vitamin D and high parathyroid hormone levels as determinants of loss of muscle strength and muscle mass (sarcopenia): the Longitudinal Aging Study Amsterdam. J Clin Endocrinol Metab 88, 5766-5772.

21. Park HA \& Kim SY (2013) Recent advance on vitamin D. J Korean Med Assoc 56, 310-318.

22. Looker AC, Johnson CL, Lacher DA, et al. (2011) Vitamin D Status: United States, 2001-2006. Hyattsville, MD: National Center for Health Statistics.

23. Hyppönen E \& Power C (2007) Hypovitaminosis D in British adults at age $45 \mathrm{y}$ : nationwide cohort study of dietary and lifestyle predictors. Am J Clin Nutr 85, 860-868.

24. Holick MF (2009) MrOs is D-ficient. J Clin Endocrinol Metab 94, 1092-1093.

25. Flynn MA, Nolph GB, Baker AS, et al. (1992) Aging in humans: a continuous 20 -year study of physiologic and dietary parameters. J Am Coll Nutr 11, 660-672.

26. Institute of Medicine (2011) Dietary Reference Intakes for Calcium and Vitamin D. Washington, DC: National Academies Press.

27. Baumgartner RN, Koehler KM, Gallagher D, et al. (1998) Epidemiology of sarcopenia among the elderly in New Mexico. Am J Epidemiol 147, 755-763.

28. Janssen I \& Ross R (2005) Linking age-related changes in skeletal muscle mass and composition with metabolism and disease. J Nutr Health Aging 9, 408-419.

29. Heymsfield SB, Smith R, Aulet M, et al. (1990) Appendicular skeletal muscle mass: measurement by dual-photon absorptiometry. Am J Clin Nutr 52, 214-218. 
30. Robinson S, Cooper C \& Sayer AA (2012) Nutrition and sarcopenia: a review of the evidence and implications for preventive strategies. J Aging Res 2012, 1-6.

31. Roth SM, Zmuda JM, Cauley JA, et al. (2004) Vitamin D receptor genotype is associated with fat-free mass and sarcopenia in elderly men. J Gerontol A Biol Sci Med Sci 59 , $10-15$.

32. Wang Y \& Deluca HF (2011) Is the vitamin D receptor found in muscle? Endocrinology 152, 354-363.

33. Wassner SJ, Li JB, Sperduto A, et al. (1983) Vitamin D deficiency, hypocalcaemia, and increased skeletal muscle degradation in rats. J Clin Invest 72, 102-112.

34. Bischoff-Ferrari HA, Dawson-Hughes B, Staehelin HB, et al. (2009) Fall prevention with supplemental and active forms of vitamin D: a meta-analysis of randomised controlled trials. BMJ 339, b3692.
35. Rejnmark L (2011) Effects of vitamin D on muscle function and performance: a review of evidence from randomized controlled trials. Ther Adv Chronic Dis 2, 25-37.

36. Jacques PF, Felson DT, Tucker KL, et al. (1997) Plasma 25-hydroxyvitamin $\mathrm{D}$ and its determinants in an elderly population sample. Am J Clin Nutr 66, 929-936.

37. Marantes I, Achenbach SJ, Atkinson EJ, et al. (2011) Is vitamin D a determinant of muscle mass and strength? J Bone Miner Res 26, 2860-2871.

38. Shantavasinkul PC, Phanachet $\mathrm{P}$, Puchaiwattananon $\mathrm{O}$, et al. (2014) Vitamin D status is a determinant of skeletal muscle mass in obesity according to body fat percentage. Nutrition 31, 801-806.

39. Bahanushali AA, Lajpal N, Kulkarni SS, et al. (2009) Frequency of fokI and taqI polymorphism of vitamin $\mathrm{D}$ receptor gene in Indian population and its association with 25-hydroxyvitamin D levels. Indian J Hum Genet 15, 108-113. 